\title{
Symmetric Integration Rules for Hypercubes I. Error Coefficients
}

\author{
By J. N. Lyness
}

Abstract. A compact notation is introduced to describe and systematise symmetric integration rules and the Euler-Maclaurin expansion is used to describe their error terms. The application to cytolic rules is discussed especially in relation to the number of function evaluations required. This paper is devoted exclusively to theory, illustrated by well-known results. This theory leads to new powerful integration rules which will be published shortly.

1. Notation. We consider in this paper symmetric integration rules for obtaining numerical approximations to the integral of a function of $n$ variables over an $n$ dimensional hypercube. There exist a large number of such rules. In this section we introduce a compact notation to describe them. We describe the notation in one dimension first.

We suppose that we are interested in the integral of the function $f(x)$ between the limits $x=-a$ and $x=a$. We term this integral If. We also define a family of basic integration rules $R(\alpha)$. Each $R(\alpha)$ is an operator which, when applied to a function $f(x)$, results in the rule which assigns equal weight to the coordinates $x=-\alpha a$ and $x=\alpha a$. Thus we may write

$$
\text { If }=\int_{a}^{a} f(x) d x
$$

and

$$
R(\alpha) f=a\{f(-\alpha a)+f(\alpha a)\} .
$$

It is trivial to show that both $I$ and $R(\alpha)$ are linear operators.

All symmetric integration rules may be considered as the weighted sum of basic rules $R(\alpha)$. For example, Simpson's rule $R_{\mathrm{B}}$ may be written

$$
R_{\mathrm{B}}=\frac{2}{3} R(0)+\frac{1}{3} R(1)
$$

and it is easy to verify by direct substitution of (1.2) into (1.3) that

$$
R_{\mathrm{B}} f=\frac{a}{3}\{f(-a)+4 f(0)+f(a)\} .
$$

This integration rule is fully defined by the linear operator $R_{B}$ in (1.3). Henceforth we shall use the term "integration rule" to denote the linear operator which generates the rule in question.

We term an integration rule $R$, such as $R_{\mathrm{g}}$ above, which is not itself a basic rule, a composite rule. All composite rules may be written in the form

$$
R=\sum \xi_{j} R\left(\alpha_{j}\right),
$$

Received May 6, 1964. This research was supported in part by U. S. Air Force Grant No. 62-400 to the University of New South Wales. 
where the $\xi_{j}$ and $\alpha_{j}$ are uniquely defined by $R, \dagger$ and vice versa. We consider in this paper only those rules which integrate a constant function exactly. Thus, if $f(x)=$ 1, we have to ensure that $R f=I f$. This leads to a condition on the $\xi_{j}$ of (1.5), namely,

$$
1=\sum \xi_{j}
$$

We now generalise these definitions to $n$ dimensions. We are interested here in integration over a hypercube $H$ of side $2 a$, bounded by the planes $x_{i}= \pm a$ $(i=1,2, \cdots, n)$. We define If as the true integral of $f\left(x_{1}, x_{2}, \cdots, x_{n}\right)$ over the hypercube $H$. Thus

$$
\text { If }=\int_{H} f(\mathbf{x}) d^{n} x .
$$

As in one dimension, we define a family of basic rules $R\left(\alpha_{1} \alpha_{2} \cdots \alpha_{n}\right)$. This rule assigns an equal weight to each of a symmetrically situated set of points. This set includes the point $\left(\alpha_{1} a, \alpha_{2} a, \cdots, \alpha_{n} a\right)$ and any point obtainable from this point by permuting the coordinates and/or reversing the sign of any or all of the coordinates. For example, the two-dimensional rule $R(\alpha, \beta)$ may be expressed as

$$
\begin{aligned}
\mathcal{R}(\alpha, \beta) f= & \frac{(2 a)^{2}}{8}\{f(\alpha a, \beta a)+f(-\alpha a, \beta a)+f(\alpha a,-\beta a)+f(-\alpha a,-\beta a) \\
& +f(\beta a, \alpha a)+f(-\beta a, \alpha a)+f(\beta a,-\alpha a)+f(-\beta a,-\alpha a)\} .
\end{aligned}
$$

This requires eight function evaluations. If one of $\alpha$ or $\beta$ is zero, or if $\alpha=\beta$, the expression contains only four terms while, using (1.8), we find

$$
R(0,0) f=(2 a)^{2} f(0,0) \text {. }
$$

In $n$ dimensions the definition is a straightforward generalisation of (1.8). In terms of the $n$ ! permutations

$$
P=\left(\begin{array}{cccc}
1 & 2 & \cdots & n \\
P_{1} & P_{2} & \cdots & P_{n}
\end{array}\right)
$$

we write

$$
\begin{aligned}
& \mathcal{R}\left(\alpha_{1} \alpha_{2} \cdots \alpha_{n}\right) f \\
& \quad=\frac{a^{n}}{n !} \sum_{\lambda_{1}= \pm 1} \sum_{\lambda_{2}= \pm 1} \cdots \sum_{\lambda_{n}= \pm 1} \sum_{P} f\left(\lambda_{1} \alpha_{P_{1}} a, \lambda_{2} \alpha_{P_{2}} a, \cdots, \lambda_{n} \alpha_{P_{n}} a\right) .
\end{aligned}
$$

Each basic rule $R\left(\alpha_{1} \alpha_{2} \cdots \alpha_{n}\right)$ is defined by a set $\left(\alpha_{1} \alpha_{2} \cdots \alpha_{n}\right)$ of non-negative coefficients $\alpha$. Any permutation of these coefficients $\alpha$ defines the same operator $R\left(\alpha_{1} \alpha_{2} \cdots \alpha_{n}\right)$.

As in the case in one dimension, any $n$-dimensional integration rule $R$, having the same symmetry as the basic rules, may be expressed as a weighted sum of them. The most general composite rule is defined by the operator

$$
R=\sum_{j} \xi_{j} R\left(\alpha_{1}{ }^{(j)} \alpha_{2}{ }^{(j)} \cdots \alpha_{n}{ }^{(j)}\right),
$$

$\dagger$ We make the obvious restriction $\alpha_{j} \neq \alpha_{k}$ for $j \neq k$. 
with the condition

$$
\sum_{j} \xi_{j}=1 \text {. }
$$

This condition ensures that the integral of $f(\mathbf{x})=1$ is given correctly by (1.12). The decomposition (1.12) is unique if we make the notational restrictions

$$
0 \leqq \alpha_{1}^{(j)} \leqq \alpha_{2}^{(j)} \leqq \cdots \leqq \alpha_{n}{ }^{(j)} \quad(\text { all } j),
$$

and there exists for all $j \neq k$ a subscript $i$ such that

$$
\alpha_{i}{ }^{(j)} \neq \alpha_{i}{ }^{(k)}, \quad j \neq k .
$$

We now define the convolution product of two basic rules; this is another basic rule defined by

$$
R\left(\alpha_{1} \alpha_{2} \cdots \alpha_{r}\right) * R\left(\beta_{1} \beta_{2} \cdots \beta_{s}\right)=R\left(\alpha_{1} \alpha_{2} \cdots \alpha_{r} \beta_{1} \beta_{2} \cdots \beta_{s}\right) .
$$

Thus the convolution product of an $r$-dimensional and $s$-dimensional basic rule is an integration rule in $r+s$ dimensions. Remembering that the order of the parameters that define a basic rule is immaterial, we see that this product is commutative and associative.

To avoid later confusion it should be emphasised that the convolution product rule operator is not simply the product of two lower-dimensional rule operators, but is a normalised symmetric sum of many such products. We write, for an $n$ dimensional operator, $n$ subscripts which indicate the dimension in which they operate. Thus

$$
\Re_{y}(\beta) f(x, y)=a\{f(x, \beta a)+f(x,-\beta a)\}
$$

and

$$
\begin{aligned}
& \mathbb{R}_{x}(\alpha) \cdot \mathbb{R}_{y}(\beta) f(x, y) \\
& \quad=a^{2}\{f(\alpha a, \beta a)+f(-\alpha a, \beta a)+f(+\alpha a,-\beta a)+f(-\alpha a,-\beta a)\} .
\end{aligned}
$$

This is not the same as $R(\alpha) * R(\beta)$, which is given by (1.8) above. We see that

$$
\begin{aligned}
\mathfrak{R}(\alpha) * \mathbb{R}(\beta) f(x, y) & =\mathbb{R}(\alpha, \beta) f(x, y) \\
& =\frac{1}{2}\left\{\mathbb{R}_{x}(\alpha) \cdot \mathfrak{R}_{y}(\beta) f(x, y)+\mathfrak{R}_{x}(\beta) \cdot \mathfrak{R}_{y}(\alpha) f(x, y)\right\}
\end{aligned}
$$

in agreement with (1.8).

The $n$-dimensional convolution product of the $(n-1)$-dimensional rule

$$
\mathbb{R}^{(n-1)}=R\left(\alpha_{1} \alpha_{2} \cdots \alpha_{n-1}\right)
$$

and the one-dimensional rule $\mathcal{R}^{(1)}=\mathscr{R}\left(\alpha_{n}\right)$ is given by

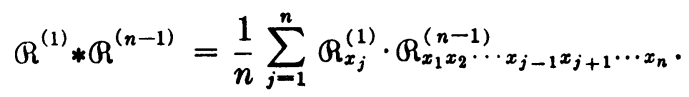

We use only the convolution product in the remainder of this paper and make no further use of subscripts to indicate the dimension in which a rule operates.

As mentioned above, any symmetric rule $R$ may be expressed as a sum of basic rules. We write

$$
R=\sum \xi_{j} \mathcal{R}_{j}
$$


and

$$
S=\sum \zeta_{k} R_{k} .
$$

We define the convolution product of $R$ and $S$ as the integration rule

$$
T=R * S=\left(\sum \xi_{j} \mathbb{R}_{j}\right) *\left(\sum \zeta_{k} \mathfrak{R}_{k}\right)=\sum \sum \xi_{j} \zeta_{k} \mathfrak{R}_{j} * \mathfrak{R}_{k},
$$

the product $R_{j} * R_{k}$ having been defined in (1.14). It is easy to show that $T$ is, indeed, an integration rule and that the product of composite rules is commutative, associative and distributive. That is, for three rules $R_{1}, R_{2}$ and $R_{3}$,

$$
\begin{aligned}
\left(R_{1} * R_{2}\right) f & =\left(R_{2} * R_{1}\right) f, \\
{\left[\left(R_{1} * R_{2}\right) * R_{3}\right] f } & =\left[R_{1} *\left(R_{2} * R_{3}\right)\right] f, \\
{\left[R_{1} *\left(\xi_{2} R_{2}+\xi_{3} R_{3}\right)\right] f } & =\left(\xi_{2} R_{1} * R_{2}\right) f+\left(\xi_{3} R_{1} * R_{3}\right) f,
\end{aligned}
$$

so long as, in the third case, $R_{2}$ and $R_{3}$ are of the same dimension.

Cases of particular interest are the conventionally termed $n$-dimensional product rules

$$
(R)^{n}=R * R * \cdots * R .
$$

For example, if $R$ is Simpson's rule $R_{\S}$ given by equation (1.3), then the threedimensional Product Simpson rule $R_{\mathrm{PS} 3}$ is

$$
\begin{aligned}
R_{\mathrm{PB} 3}=\left(R_{\mathrm{B}}\right)^{3} & =\left(\frac{2}{3} R(0)+\frac{1}{3} \mathcal{R}(1)\right)^{3} \\
& =\frac{8}{27} \mathcal{2}(0,0,0)+\frac{1}{2} \frac{2}{7} \mathcal{R}(1,0,0)+\frac{8}{27} \mathcal{R}(1,1,0)+\frac{1}{27} R(1,1,1) .
\end{aligned}
$$

However, there are many $n$-dimensional rules which are not product rules (though all are weighted sums of product rules). These include, for example, the threedimensional centre and vertex rule

$$
R_{\mathrm{CV} 3}=\frac{2}{3} R(0,0,0)+\frac{1}{3} R(1,1,1) .
$$

2. The Error Expansion in One Dimension. In this section we investigate what we term the 'error' involved in using a particular rule $R$. By the convenient term 'error' we refer to the difference between the exact integral If and the exactly calculated approximation $R(\alpha) f$. We do not include in this paper any discussion of rounding or truncation errors. We deal first with the one-dimensional basic rule $R(\alpha)$. An adaption of the Euler-Maclaurin expansion leads to an expansion of the error $R(\alpha) f-I f$ in terms of the integrals of the even order derivatives of $f$. This expansion is given in (2.7) below. We outline an elementary derivation of (2.7) here. We may expand various quantities in power series as follows:

$$
\begin{aligned}
\Re(\alpha) f & =a(f(-\alpha a)+f(\alpha a)) \\
& =2 a\left\{f(0)+\frac{a^{2} \alpha^{2}}{2 !} f^{\prime \prime}(0)+\frac{a^{4} \alpha^{4}}{4 !} f^{(\mathrm{iv})}(0)+\cdots\right\}
\end{aligned}
$$

and

$$
I f=\int_{-a}^{a} f(x) d x=2 a\left\{f(0)+\frac{a^{2}}{3 !} f^{\prime \prime}(0)+\frac{a^{4}}{5 !} f^{(\mathrm{iv})}(0)+\cdots\right\} .
$$


We eliminate $f(0)$ from these equations to find

$$
\left.\mathcal{R}(\alpha) f-I f=2 a\left\{\left(\frac{\alpha^{2}}{2 !}-\frac{1}{3 !}\right) a^{2} f^{\prime \prime}(0)+\left(\frac{\alpha^{4}}{4 !}-\frac{1}{5 !}\right) a^{4} f^{(\mathrm{iv})}(0)-\ldots\right\}\right\} .
$$

If we write down $(2.2)$ with $f^{(2 s)}(x)$ replacing $f(x)$, we find

$$
\int_{-a}^{a} f^{(2 s)}(x) d x=2 a\left\{f^{(2 s)}(0)+\frac{a^{2}}{3 !} f^{(2 s+2)}(0)+\cdots\right\} ;
$$

eliminating $f^{\prime \prime}(0)$ from (2.3) and (2.4) with $8=1$ leads to

$$
\begin{aligned}
R(\alpha) f-I f= & a^{2}\left(\frac{\alpha^{2}}{2 !}-\frac{1}{3 !}\right) \int_{a}^{a} f^{\prime \prime}(x) d x \\
& \quad+2 a\left\{\left\{\left(\frac{\alpha^{4}}{4 !}-\frac{1}{5 !}\right)-\frac{1}{3 !}\left(\frac{\alpha^{2}}{2 !}-\frac{1}{3 !}\right)\right\} a^{4} f^{(\text {iv })}(0)+\cdots\right\} .
\end{aligned}
$$

Elimination of $f^{(\mathrm{iv})}(0)$ between (2.5) and (2.4) with $8=2$ gives explicitly the first two terms of the expansion, namely

$$
\begin{aligned}
\mathcal{R}(\alpha) f-I f=\frac{a^{2}}{2 !}\left\{-\frac{1}{3}+\alpha^{2}\right\} \int & f^{\prime \prime}(x) d x \\
+ & \frac{a^{4}}{4 !}\left\{\frac{7}{15}-2 \alpha^{2}+\alpha^{4}\right\} \int f^{(\text {iv })}(x) d x+\cdots .
\end{aligned}
$$

This procedure may be continued indefinitely. The expansion takes the form

$$
R(\alpha) f-I f=\sum_{s=1}^{\infty} a^{2 s} c_{2 s}(\alpha) \int_{-a}^{a} f^{(2 s)}(x) d x,
$$

where $c_{2 s}(\alpha)$ are functions of $\alpha$, two of which are explicitly determined in (2.6). For later convenience we define

$$
c_{0}(\alpha)=1 .
$$

The coefficients $c_{2 s}(\alpha)$ may be determined most easily by using a generating function technique. We insert the function

$$
f(x)=\frac{\varphi}{a} \cosh \frac{\varphi x}{a}
$$

into (2.7) and carry out the various operations analytically. This leads directly to the generating function:

$$
\sum_{r=0}^{\infty} c_{2 r}(\alpha) \varphi^{2 r}=\frac{\varphi \cosh \alpha \varphi}{\sinh \varphi} \equiv G(\alpha, \varphi) .
$$

This generating function may be used to find $c_{2 s}(0)$ and $c_{2 s}(\alpha)$ for certain rational values, in particular, $c_{2 s}(1)$ and $c_{2 s}(1 / 2) . c_{28}(0)$ is the coefficient of $\varphi^{2 s}$ in the power expansion of $\varphi \operatorname{cosech} \varphi$. This gives the following value, in terms of the Bernoulli numbers.

$$
c_{28}(0)=(-1)^{r}\left(2^{2 r}-2\right) B_{2 r-1} /(2 r) !
$$


We also find directly from the generating function that

$$
c_{28}(1 / 2)=c_{2 s}(0) / 2^{2 r}
$$

and

$$
c_{2 s}(1)=-c_{2 s}(0) /\left(1-2^{1-2 r}\right) .
$$

To find $c_{2 s}(\alpha)$ we rewrite the generating function $(2.10)$ in the form

$$
\sum c_{2 r}(0) \varphi^{2 r} \cosh \alpha \varphi=\sum c_{2 r}(\alpha) \varphi^{2 r} .
$$

The coefficient of $\varphi^{2 r}$ in this leads to an expression for $c_{2 s}(\alpha)$ in terms of $\alpha$ and $c_{2 t}(0)$ with $t \leqq s$, namely

$$
c_{2 s}(\alpha)=c_{2 s}(0)+\frac{\alpha^{2}}{2 !} c_{2 s-2}(0)+\frac{\alpha^{4}}{4 !} c_{2 s-4}(0)+\cdots+\frac{\alpha^{2 s}}{2 s !} c_{0}(0) .
$$

Other methods (in certain cases simpler) of finding $c_{2 s}(\alpha)$ are given in the Appendix.

Using the properties of Bernoulli numbers, we may show

$$
\operatorname{Lim}_{r \rightarrow \infty}(-1)^{r} \pi^{2 r} c_{2 r}(\alpha)=2 \cos \alpha \pi,
$$

and this asymptotic form is surprisingly accurate for relatively small values of $2 r$. In fact, one may write

$$
c_{2 r}(\alpha)=\frac{2(-1)^{r}}{\pi^{2 r}}(\cos \alpha \pi+\epsilon),
$$

where $|\epsilon|<.02$ for $2 r>6$ and $|\alpha|<1$.

Using (2.11) and (2.15) above, we find

$$
\begin{aligned}
& c_{0}(\alpha)=1, \\
& c_{2}(\alpha)=\frac{1}{2 !}\left\{-\frac{1}{3}+\alpha^{2}\right\}, \\
& c_{4}(\alpha)=\frac{1}{4 !}\left\{\frac{7}{15}-2 \alpha^{2}+\alpha^{4}\right\}, \\
& c_{8}(\alpha)=\frac{1}{6 !}\left\{-\frac{31}{21}+7 \alpha^{2}-5 \alpha^{4}+\alpha^{6}\right\}, \\
& c_{8}(\alpha)=\frac{1}{8 !}\left\{\frac{127}{15}-\frac{124}{3} \alpha^{2}+\frac{98}{3} \alpha^{4}-\frac{28}{3} \alpha^{6}+\alpha^{8}\right\} .
\end{aligned}
$$

Having discussed the error term for the basic rules $\mathbb{R}(\alpha)$ in one-dimensional quadrature, we now turn to the composite rules $R$. Let us calculate the expansion of $R f-I f$. Since

$$
R=\sum \xi_{j} R\left(\alpha_{j}\right)
$$

and

$$
1=\sum \xi_{j}
$$

it appears that

$$
R f-I f=\sum \xi_{j}\left(R\left(\alpha_{j}\right) f-I f\right),
$$


and the expansion is of similar form to (2.7). We write $\nmid$

$$
c_{2 s}(R)=\sum \xi_{j} c_{2 s}\left(\alpha_{j}\right)
$$

and find

$$
R f-I f=a^{2} c_{2}(R) \int f^{(2)}(x) d x+a^{4} c_{4}(R) \int f^{(4)}(x) d x+\cdots .
$$

We simplify this expression by introducing another linear operator $\beta_{2 \triangleleft}(R)$, where

$$
\beta_{2 s}(R) f=a^{2 s} c_{2 s}(R) \int f^{(2 s)}(x) d x .
$$

The operator $\beta_{0}$ is identical to $I$ because, as a formal result of (2.8) and (2.22), we have

$$
c_{0}(\dot{R})=1
$$

(2.7) may be written

$$
R f-I f=\sum_{s=1}^{\infty} \beta_{2 s}(R) f .
$$

We note that the degree $d$ of a rule $R$ may determine some of the coefficients $c_{2 s}(R)$. If $R$ is of degree $d=2 t+1$, then $R f-I f$ is zero whenever $f$ is a polynomial of degree less than or equal to $d$. We consider in turn $f=x^{2}, x^{4}, \cdots, x^{(d-1)}$ in (2.26) above. These give $d$ linear equations for $c_{2 s}(R)$ which have the simple solution

$$
c_{2 s}(R)=0, \quad 1<2 s<d .
$$

The construction of high-degree rules may be effected by substituting into this equation the expansion (2.22). The equations so found are nonlinear simultaneous equations and are simply related to those obtained by requiring the appropriate integrals to be fitted exactly.

3. The $n$-Dimensional Error Expansion. In $n$ dimensions the general procedure is similar to that in one dimension. The generalisation of the expansion (2.7) may be obtained in an analogous way. We may make the $n$-dimensional Taylor expansion

$$
\begin{aligned}
\Re\left(\alpha_{1} \alpha_{2} \cdots \alpha_{n}\right) f= & (2 a)^{n} f(0,0, \cdots, 0) \\
& +(2 a)^{n} \sum_{r=1}^{\infty} a^{2 r} \sum_{\Sigma s_{i}=r} d_{2 s_{1} 2 s_{2} \cdots 2 s_{n}}\left[\frac{\partial^{2 r} f}{\partial x_{1}{ }^{2 s_{1}} \partial x_{2}{ }^{2 s_{2}} \cdots \partial x_{n}{ }^{2 s_{n}}}\right]_{0},
\end{aligned}
$$

the subscript zero on the square bracket indicating that the partial derivative is evaluated at the point $(0,0, \cdots, 0)$; the summation over $s_{i}$ includes all sets of non-negative integers $s_{i}$ whose sum is $r$.

We also use the expansion

$$
\begin{aligned}
(2 a)^{n} a^{2 r} & {\left[\frac{\partial^{2 r} f}{\partial x_{1}^{2 s_{1}} \partial x_{2}^{2 s_{2}} \cdots \partial x_{n}{ }^{2 s_{n}}}\right]_{0}=a^{2 r} \int_{B} \frac{\partial^{2 r f}}{\partial x_{1}^{2 s_{1}} \partial x_{2}^{2 s_{2}} \cdots \partial x_{n}^{2 s_{n}}} d^{n} x } \\
& -(2 a)^{n} \sum_{s=1}^{\infty} \sum_{\Sigma t_{i}=s} e_{2 t_{1} 2 t_{2} \cdots 2 t_{n}} a^{2 r+2 s}\left[\frac{\partial^{2 r+2 s f}}{\partial x_{1}^{2 s_{1}+2 t_{1}} \partial x_{2}^{2 s_{2}+2 t_{2}} \cdots \partial x_{n}^{2 s_{n}+2 t_{n}}}\right]_{0} .
\end{aligned}
$$

† For notational convenience, $c_{2 s}(\alpha)$ is written for $c_{2 s}(R(\alpha))$. These numbers are referred to as error coefficients. 
These expansions are the generalisations of (2.1) and (2.4). The coefficients $d$ and $e$ are numbers which may depend on $\alpha_{1}, \alpha_{2}, \cdots, \alpha_{n}$, but not on $a$ or $f$.

We now carry out the same procedure that we have already described in Section 2. We use (3.2) with $r=0$ to eliminate $f_{0}$ from (3.1). We then use (3.2) with $r=1$ and different choices of $s_{i}$ to eliminate the second-order derivatives in the resulting expression. We may carry on this elimination indefinitely to obtain

$$
\Re f-I f=\sum_{s=1}^{\infty} a^{2 s} \sum_{\Sigma s_{i}=8} c_{2 s_{1} 2 s_{2} \cdots 2 s_{n}}(R) \int_{H} \frac{\partial^{2 s f}}{\partial x_{1}^{2 s_{1}} \partial x_{2}^{2 s_{2}} \cdots \partial x_{n}^{2 s_{n}}} d^{n} x,
$$

where the error coefficients $c_{28_{1} 2 s_{2} \cdots 2 s_{n}}(R)$ depend only on the parameters which define $R$, namely, $\alpha_{1}, \alpha_{2}, \cdots, \alpha_{n}$.

We define the operator $\beta_{28}(R)$ as follows:

$$
\beta_{2 s}(R) f=a^{2 s} \sum_{\Sigma s_{j}=8} c_{2 s_{1} 2 s_{2} \cdots 2 s_{n}}(R) \int_{H} \frac{\cdot \partial^{2 s f}}{\partial x_{1}^{2 s_{1}} \partial x_{2}^{2 s_{2}} \cdots \partial x_{n}^{2 s_{n}}} d^{n} x .
$$

For the purpose of being quite explicit, we write out in full the first few $\beta_{2 s}(R)$. These are

$$
\begin{aligned}
& \beta_{2}(R) f=a^{2} c_{200 \ldots} \ldots(R) \int_{H} \sum_{i=1}^{n} \frac{\partial^{2} f}{\partial x_{i}{ }^{2}} d^{n} x \\
& \beta_{4}(R) f=a^{4} c_{400 \ldots}(R) \int_{B} \sum_{i=1}^{n} \frac{\partial^{4} f}{\partial x_{i}{ }^{4}} d^{n} x+a^{4} c_{220 \ldots}(R) \int_{H} \sum_{i=1}^{n} \sum_{j=1 ; j \neq i}^{n} \frac{\partial^{4} f}{\partial x_{i}^{2} \partial x_{j}{ }^{2}} d^{n} x, \\
& \beta_{6}(\Re) f=a^{6} c_{600} \ldots(R) \int_{H} \sum_{i=1}^{n} \frac{\partial^{6} f}{\partial x_{i}{ }^{6}} d^{n} x+a^{6} c_{420} \ldots(R) \int_{H} \sum_{i=1}^{n} \sum_{j=1}^{n} \frac{\partial^{6} f}{\partial x_{i}{ }^{4} \partial x_{j}{ }^{2}} d^{n} x \\
& +a^{6} c_{222 \ldots} \ldots \text { (R) } \int_{H} \sum_{i=1}^{n} \sum_{j=1 ; j \neq i}^{n} \sum_{k=1 ; k \neq i ; k \neq j}^{n} \frac{\partial^{6} f}{\partial x_{i}^{2} \partial x_{j}^{2} \partial x_{k}^{2}} d^{n} x \text {. }
\end{aligned}
$$

The number of subscripts attached to each error coefficient is the same as the number of dimensions. Thus, if $n=2$, the third group of terms in $\beta_{6}(\mathcal{R}) f$, i.e., those with coefficient $c_{222}(R)$, does not exist. The number of separate terms in $\beta_{28}(R)$ in $n$ dimensions is $(n+s-1) ! /(s !(n-1) !)$.

The expansion (3.3) may now be written

$$
\text { Rf }-I f=\sum_{s=1}^{\infty} \beta_{2 s}(R) f,
$$

which is formally exactly the same as in the one-dimensional case (2.26), the difference being in the generalisation of the operators $R, I$ and $\beta_{2 s}$.

We now determine the coefficients $c_{2 s_{1} 2 \varepsilon_{2} \cdots 2 s_{n}}(R)$ corresponding to the basic rule $\mathcal{R}\left(\alpha_{1} \alpha_{2} \cdots \alpha_{n}\right)$. We write in (3.3) the function:

$$
f(\mathbf{x})=\left[\frac{\varphi_{1}}{a} \cosh \frac{\varphi_{1} x_{1}}{a}\right]\left[\frac{\varphi_{2}}{a} \cosh \frac{\varphi_{2} x_{2}}{a}\right] \cdots\left[\frac{\varphi_{n}}{a} \cosh \frac{\varphi_{n} x_{n}}{a}\right] .
$$

Carrying out all the implied differentiation and integration, we find

$$
\begin{array}{r}
\sum_{s=1}^{\infty} \sum_{\Sigma s_{j}=s} c_{2 s_{1} 2 s_{2} \cdots 2 s_{n}} \varphi_{1}^{2 s_{1}} \varphi_{2}^{2 s_{2}} \cdots \varphi_{n}^{2 s_{n}} \sinh \varphi_{1} \sinh \varphi_{2} \cdots \sinh \varphi_{n} \\
=\frac{1}{n !} \sum_{P} \varphi_{1} \cosh \alpha_{P_{1}} \varphi_{1} \varphi_{2} \cosh \alpha_{P_{2}} \varphi_{2} \cdots \varphi_{n} \cosh \alpha_{P_{n}} \varphi_{n}
\end{array}
$$

$-\sinh \varphi_{1} \sinh \varphi_{2} \cdots \sinh \varphi_{n}$. 
The summation symbol on the right of this equation was defined after equation (1.10). Rearrangement of this expression and introduction of the one-dimensional generating function $G(\alpha, \varphi)$ of $(2.10)$ leads to

$$
\begin{aligned}
\sum_{i=0}^{\infty} \sum_{\Sigma s_{j}=t} c_{2 s_{1} 2 s_{2} \cdots 2 \delta_{n}} \varphi_{1}{ }^{2 s_{1}} \varphi_{2}{ }^{2 s_{2}} & \cdots \varphi_{n}{ }^{2 \delta_{n}} \\
= & +\frac{1}{n !} \sum_{P} G\left(\alpha_{P_{1}}, \varphi_{1}\right) G\left(\alpha_{P_{2}}, \varphi_{2}\right) \cdots G\left(\alpha_{P_{n}}, \varphi_{n}\right),
\end{aligned}
$$

with the convention

$$
c_{00 \cdots 0}(R)=+1 \text {. }
$$

Inserting the expansion for $G(\alpha, \varphi)$ in terms of the one-dimensional coefficients $c_{28}(\alpha)$, and considering the coefficient of $\varphi_{1}^{2 s_{1}} \varphi_{2}^{2 s_{2}} \cdots \varphi_{n}{ }^{2 s_{n}}$, we find

$$
c_{2 \alpha_{1} 2 \alpha_{2} \cdots 2 s_{n}}\left(R\left(\alpha_{1} \alpha_{2} \cdots \alpha_{n}\right)\right)=\frac{1}{n !} \sum_{P} c_{2 \alpha_{1}}\left(\alpha_{P_{1}}\right) c_{2 \alpha_{2}}\left(\alpha_{P_{3}}\right) \cdots c_{2 \alpha_{n}}\left(\alpha_{P_{n}}\right),
$$

where the subscript $P$ indicates (as in the definition in (1.11)) that we sum over the $n$ ! terms obtained by replacing the numbers $P_{1} P_{2} \cdots P_{n}$ by the $n !$ permutations of $1,2, \cdots, n$ in turn.

Equation (3.13) may be rewritten

$$
c_{2 s_{1} 2 \varepsilon_{2} \cdots 2 s_{n}}\left(R\left(\alpha_{1} \alpha_{2} \cdots \alpha_{n}\right)\right)=\frac{1}{n !} \sum_{P} c_{2 q_{1}}\left(\alpha_{1}\right) c_{2 q_{2}}\left(\alpha_{2}\right) \cdots c_{2 q_{n}}\left(\alpha_{n}\right),
$$

where $q_{i}=s_{P_{i}}$.

That is to say, we may permute the subscripts $s_{1}, s_{2}, \cdots, s_{n}$ instead of the parameters $\alpha_{1}, \alpha_{2}, \cdots, \alpha_{n}$. Since we sum over all the permutations, (3.14) is simply a reordering of (3.13).

As an example of this formula, we apply it to the face centre rule in three dimensions $R_{\mathrm{rC} 3}=R(1,0,0)$. This gives

$$
\begin{aligned}
& c_{2 \varepsilon_{1} 2 \varepsilon_{2} 2 \varepsilon_{3}}\left(R_{\mathrm{FC} 3}\right) \\
& =\frac{1}{3}\left\{c_{2 s_{1}}(1) c_{2 s_{2}}(0) c_{2 s_{3}}(0)+c_{2 s_{1}}(0) c_{2 s_{2}}(1) c_{2 s_{3}}(0)+c_{2 s_{1}}(0) c_{2 z_{2}}(0) c_{2 s_{3}}(1)\right\} .
\end{aligned}
$$

We note that $c_{200}\left(R_{\mathrm{FC} 3}\right)$ may be evaluated using equations $(2.18)$ and is zero. Thus $R(1,0,0)$ is at least of degree three.

The results extend to composite rules in a straightforward manner, in the same way as in one dimension. We write

$$
R=\sum \xi_{j} \boldsymbol{R}_{j}
$$

and

$$
1=\sum \xi_{j}
$$

Then we find, as before,

$$
R f-I f=\sum_{j} \xi_{j}(R f-I f),
$$

and so the expansion is the weighted sum of the expansion of $R f$ - If given by (3.8) above. We introduce the notation

$$
c_{2 s_{1} 2 s_{2} \cdots 2 s_{n}}(R)=\sum \xi_{j} c_{2 s_{1} 2 s_{2} \cdots 2 s_{n}}\left(R_{j}\right)
$$


and

$$
\beta_{28}(R)=\sum \xi_{j} \beta_{28}\left(R_{j}\right)
$$

This gives at once

$$
\begin{aligned}
R f-I f & =\sum_{s=1}^{\infty} a^{2 s} \sum_{\Sigma s_{i}-s} c_{2 s_{1} 2 s_{2} \cdots 2 s_{n}}(R) \int_{H} \frac{\partial^{2 s} f}{\partial x_{1}{ }^{2 s_{1}} \partial x_{2}{ }^{2 s_{2}} \cdots \partial x_{n}{ }^{2 s_{n}}} d^{n} x \\
& =\sum_{s=1}^{\infty} \beta_{2 s}(R) f .
\end{aligned}
$$

The degree $d=2 t+1$ of a rule $R$ is related to the error coefficients $c_{2 s_{1} 2 s_{2} \cdots \varepsilon_{n}}(R)$ in a similar way to the one-dimensional relation (2.27). For a rule of degree $d$ it is necessary that all functions $x_{1}{ }^{28_{1}} x_{2}{ }^{28_{2}} \cdots x_{n}{ }^{2 s_{n}}$, where $\sum 2 s_{i}<d$, should be integrated exactly. By writing these functions in turn in (3.21) we find a set of linear equations in $c_{2 s_{1} 2 s_{2} \cdots 2 s_{n}}(R)$ whose solution is

$$
c_{2 s_{1} 2 s_{2} \cdots 2 s_{n}}(R)=0, \quad 1<\sum 2 s_{i}<d .
$$

An example is the $n$-dimensional corner and vertex rule $R_{\mathrm{Cv}}$ defined by

$$
R_{\mathrm{CV}}=\frac{2}{3} R(0,0, \cdots, 0)+\frac{1}{3} R(1,1, \cdots, 1) .
$$

The error coefficients $c_{28_{1} 28_{2} \cdots 2 s_{n}}\left(R_{\mathrm{CV}}\right)$ of this rule are then

$$
\begin{aligned}
c_{2 s_{1} 2 s_{2} \cdots 2 s_{n}}\left(R_{\mathrm{CV}}\right)= & \frac{2}{3} c_{2 s_{1} 2 s_{2} \cdots 2 s_{n}}(R(0,0, \cdots, 0)) \\
& +\frac{1}{3} c_{2 s_{1} 2 s_{2} \cdots 2 s_{n}}(R(1,1, \cdots, 1)) \\
= & \frac{2}{3} c_{2 s_{1}}(0) c_{2 s_{2}}(0) \cdots c_{2 s_{n}}(0)+\frac{1}{3} c_{2 s_{1}}(1) c_{2 s_{2}}(1) \cdots c_{2 s_{n}}(1) .
\end{aligned}
$$

We note that $c_{200 \ldots 0}\left(R_{\mathrm{CV}}\right)=0$, and so this rule is at least of degree three.

Formula (3.13) expresses an error coefficient of the convolution product of basic rules in terms of the error coefficients of each one-dimensional constituent rule of the product. We now show that a result of this type holds for the convolution product of composite rules. We consider the product of rules $R^{(1)} R^{(2)} \cdots R^{(n)}$. Each of these may be expressed in terms of basic rules and we call the coordinates required $\alpha_{1}, \alpha_{2}, \cdots, \alpha_{t}$. Thus

$$
R^{(i)}=\sum_{j=1}^{t} \xi_{j}^{(i)} \Re\left(\alpha_{j}\right), \quad i=1, \cdots, n .
$$

In general, some, but not all, of the coordinates $\xi_{j}^{(i)}$ are zero. Using (3.24) and (3.19) we see that

$$
\begin{aligned}
c_{2 s_{1} 2 s_{2} \cdots 2 s_{n}} & \left(R^{(1)} * R^{(2)} * \cdots * R^{(n)}\right) \\
& =\sum_{j=1}^{t} \sum_{k=1}^{t} \cdots \sum_{w=1}^{t} \xi_{j}{ }^{(1)} \xi_{k}{ }^{(2)} \cdots \xi_{w}{ }^{(n)} c_{2 s_{1} 2 s_{2} \cdots 2 s_{n}}\left(\Re\left(\alpha_{j}\right) * R\left(\alpha_{k}\right) * \cdots * R\left(\alpha_{w}\right)\right) .
\end{aligned}
$$

Using (3.13) this becomes

$$
\sum_{j=1}^{t} \sum_{k=1}^{t} \cdots \sum_{w=1}^{t} \xi_{j}^{(1)} \xi_{k}{ }^{(2)} \cdots \xi_{w}{ }^{(n)} \frac{1}{n !} \sum_{P} c_{2 q_{1}}\left(\alpha_{j}\right) c_{2 q_{2}}\left(\alpha_{k}\right) \cdots c_{2 q_{n}}\left(\alpha_{w}\right),
$$

where, as before,

$$
q_{i}=s_{P_{i}}
$$


Rearrangement of the order of summation in this finite sum gives

$$
\frac{1}{n !} \sum_{P}\left(\sum_{j=1}^{t} \xi_{j}{ }^{(1)} c_{2 q_{1}}\left(\alpha_{j}\right)\right)\left(\sum_{k=1}^{t} \xi_{k}{ }^{(2)} c_{2 q_{2}}\left(\alpha_{k}\right)\right) \cdots\left(\sum_{w=1}^{t} \xi_{w}{ }^{(n)} c_{2 q_{n}}\left(c_{w}\right)\right)
$$

and this is

$$
\frac{1}{n !} \sum_{P} c_{2 q_{1}}\left(R^{(1)}\right) c_{2 q_{2}}\left(R^{(2)}\right) \cdots c_{2 q_{n}}\left(R^{(n)}\right) .
$$

Since the $q_{i}$ are a permutation of the $s_{i}$ we finally obtain

$$
c_{2 s_{1} 2 s_{2} \cdots 2 s_{n}}\left(R^{(1)} * R^{(2)} * \cdots * R^{(n)}\right)=\frac{1}{n !} \sum_{P} c_{2 s_{1}}\left(R^{\left(P_{1}\right)}\right) c_{2 s_{2}}\left(R^{\left(P_{2}\right)}\right) \cdots c_{2 s_{n}}\left(R^{\left(P_{n}\right)}\right) .
$$

This is a generalisation of (3.13) to composite rules. This formula is particularly useful in the case where $R^{(1)}, R^{(2)}, \cdots, R^{(n)}$ are all the same rule $R$. We find

$$
c_{2 s_{1} 2 s_{2} \cdots 2 s_{n}}\left(R^{n}\right)=c_{2 s_{1}}(R) c_{2 s_{2}}(R) \cdots c_{2 s_{n}}(R) .
$$

Using this equation it is trivial to verify that if $R$ is of degree $d$ so is $R^{n}$. The error coefficients of the Product Simpson rule

$$
R_{\mathrm{PB}}=\left(\frac{2}{3} R(0)+\frac{1}{3} R(1)\right)^{n}
$$

are given by

$$
\begin{aligned}
& c_{2 s_{1} 2 s_{2} \cdots 2 s_{n}}\left(R_{\mathrm{Pg}}\right) \\
& \quad=\left(\frac{2}{3} c_{2 s_{1}}(0)+\frac{1}{3} c_{2 s_{1}}(1)\right)\left(\frac{2}{3} c_{2 s_{2}}(0)+\frac{1}{3} c_{2 s_{2}}(1)\right) \cdots\left(\frac{2}{3} c_{2 s_{n}}(0)+\frac{1}{3} c_{2 s_{n}}(1)\right) .
\end{aligned}
$$

This calculation is more straightforward than expanding the product rule and determining the coefficients of each term separately. That would lead to an expression, whose value is identical to (3.28), but fully expanded as the sum of $2^{n}$ products, each of $n$ terms.

4. Cytolic Integration. In the practice of numerical integration it is often convenient or advantageous to divide the region of integration into small hypercubic cells and to use a simple integration rule in each. (See, for example, Miller [3] (1960), or Mustard, Lyness and Blatt [5] (1963).) $\dagger$

We suppose that the hypercube $H$ defined by $\left|x_{i}\right|<a(i=1, \cdots, n)$ has been divided up into $r^{n}$ equal hypercubic subdomains $H_{j}^{(r)}\left(j=1,2, \cdots, r^{n}\right)$, each having side length

$$
2 h=2 a / r \text {. }
$$

We may use the same rule $R$ in each subdomain and obtain the final integral by adding up the $r^{n}$ contributions so obtained. This, of course, corresponds to using a much more complicated rule $R^{(r)}$ over the whole hypercube $H$. We introduce the

† The terms cytolic and holistic were introduced by Mustard [6] (1964). Cytolic (Greek: $\operatorname{kuros}^{\prime}=$ vessel) is used to describe integration by division into cells, and holistic (Greek: $\dot{\partial} \mathrm{\lambda os}=$ whole) to describe integration treating the whole domain at once. An equivalent definition appears to be that, with the exception of the above-mentioned references, holistic integration refers to the integration techniques discussed in the literature and cytolic integration to techniques used in practice. 
notation $I_{j}^{(r)} f$ for the integral of $f$ over the hypercube $H_{j}^{(r)}$ and $R_{j}^{(r)} f$ for the numerical approximation to $I_{j}^{(r)} f$ obtained by applying rule $R$ to this hypercube.

We illustrate this notation in one dimension. Simpson's rule $R_{\mathbb{8}}$ has the form

$$
R_{\mathrm{B}} f=\left(\frac{2}{3} R(0)+\frac{1}{3} R(1)\right) f=2 a\left\{\frac{1}{6} f(-a)+\frac{2}{3} f(0)+\frac{1}{6} f(a)\right\} .
$$

In practice, it is by no means unusual to break the region of integration up into $r$ equal smaller regions and use Simpson's rule on each. Thus we might write, in the case $r=3$,

$$
\begin{aligned}
& R_{\mathrm{B}}{ }^{(3)} f \\
& =\frac{2 a}{3}\left\{\frac{1}{6} f(-a)+\frac{2}{3} f\left(-\frac{2}{3} a\right)+\frac{1}{3} f\left(-\frac{1}{3} a\right)+\frac{2}{3} f(0)+\frac{1}{3} f\left(\frac{1}{3} a\right)+\frac{2}{3} f\left(\frac{2}{3} a\right)+\frac{1}{6} f(a)\right\} .
\end{aligned}
$$

This is the sum of three expressions, the first of which is

$$
R_{1}{ }^{(3)} f=\frac{2 a}{3}\left\{\frac{1}{8} f(-a)+\frac{2}{3} f\left(-\frac{2}{3} a\right)+\frac{1}{6} f\left(-\frac{1}{3} a\right)\right\} .
$$

Moreover, $R_{\mathrm{s}}{ }^{(3)}$ is expressible as the sum of basic rules. We see that

$$
R_{\mathrm{B}}{ }^{(3)}=\frac{1}{9} R(1)+\frac{4}{9} R\left(\frac{2}{3}\right)+\frac{2}{9} R\left(\frac{1}{3}\right)+\frac{2}{9} R(0) .
$$

As rules such as $R^{(r)}$ are in frequent use it is of interest to calculate their error coefficients. Calculated in the straightforward way by means of such expressions as (4.5) above, this could be quite lengthy. In fact, of course, the error coefficients of a rule $R^{(r)}$ are simply related to the error coefficients of $R$. We determine this relation below. We may write, for the error,

$$
R^{(r)} f-I f=\sum_{i=1}^{r^{n}}\left(R_{j}^{(r)}-I_{j}^{(r)}\right) f .
$$

The expansion of $R_{j}^{(r)} f-I_{j}^{(r)} f$ is given in Section 3 by (3.21) if we replace $a$ by $h$ and use the appropriate region of integration $H_{j}$. We find:

$$
R_{j}^{(r)} f-I_{j}^{(r)} f=\sum_{s=1}^{\infty} h^{2 s} \sum_{\Sigma s_{i}=s} c_{2 s_{1} 2 s_{2} \cdots s_{n}}(R) \int_{H_{j}} \frac{\partial^{2 s} f}{\partial x_{1}^{2 s_{1}} \partial x_{2}^{2 s_{2}} \cdots \partial x_{n}{ }^{2 s_{n}}} d^{n} x
$$

We obtain the total error by summing (4.7) with $j=1,2, \cdots, r^{n}$. Since $j$ occurs on the right-hand side only in the definition of the region of integration $H_{j}$ and not in the integrand, and these regions are distinct and combine to form $H$, we find

$$
R^{(r)} f-I f=\sum_{s=1}^{\infty} h^{2 s} \sum_{\Sigma s_{i}=s} c_{2 s_{1} 2 s_{2} \cdots 2 s_{n}}(R) \int_{H} \frac{\partial^{2} f}{\partial x_{1}^{2 s_{1}} \partial x_{2}^{2 s_{2}} \cdots \partial x_{n}^{2 s_{n}}} d^{n} x .
$$

This error expansion is almost identical to the expansion of $R f$ - If given by (3.3), the only difference being that $h^{2 s}$ occurs instead of $a^{2 s}$ on the left-hand side. Thus we may make use of the operators $\beta_{2 s}(R)$ of (3.20) to obtain

$$
R^{(r)} f-I f=\sum_{s=1}^{\infty} \frac{1}{r^{2 s}} \beta_{28}(R) f
$$


This expression is the basis of the "Progressive Procedure" (Lyness and McHugh [2] (1963)). The required relations are simply

$$
\beta_{2 s}\left(R^{(r)}\right)=\frac{1}{r^{2 s}} \beta_{2 s}(R)
$$

and

$$
c_{2 s_{1} 2 s_{2} \cdots 2 s_{n}}\left(R^{(r)}\right)=\frac{1}{r^{28}} c_{2 s_{1} 2 s_{2} \cdots 2 s_{n}}(R) .
$$

5. The Number of Function Evaluations. One of the goals of the analysis of integration rules is to determine which rule requires fewest function evaluations to obtain a result of particular accuracy or degree. Thus an. important property of any integration rule $R$ is the number of points at which it is necessary to evaluate the function. If the rule is used over a single hypercubic domain to obtain $R f$ or $R_{j}^{(r)} f$ we define $\nu_{1}(R)$ to be this number.

It is straightforward to determine the number of points used by a basic rule. If

$$
R=R(0)^{n_{0}} * R\left(\alpha_{1}\right)^{n_{1}} * R\left(\alpha_{2}\right)^{n_{2}} * \cdots * R\left(\alpha_{k}\right)^{n_{k}},
$$

where

$$
n_{0}+n_{1}+n_{2}+\cdots+n_{k}=n
$$

and

then

$$
0<\alpha_{1}<\alpha_{2} \cdots<\alpha_{k}
$$

$$
\nu_{1}(R)=\frac{n !}{n_{0} ! n_{1} ! n_{2} ! \cdots n_{k} !} 2^{n-n_{0}} .
$$

The points at which function evaluation is necessary are different for different basic rules. Thus if

$$
R=\sum_{j} \xi_{j} \mathcal{R}_{j}
$$

it follows that

$$
\nu_{1}(R)=\sum_{j} \nu_{1}\left(R_{j}\right) .
$$

In the case of cytolic integration in which the domain of integration is divided into $r^{n}$ separate regions, the question of counting the number of points may be complicated. We define the effective number of evaluation points per subdomain as

$$
\nu_{r}(R)=\frac{1}{r^{n}} \nu_{1}\left(R^{(r)}\right) .
$$

If the rule $R$ uses only points in the interior of each cell, the total number of function evaluations required by $R^{(r)}$ is simply

$$
\nu_{1}\left(R^{(r)}\right)=r^{n} \nu_{1}(R)
$$

and, in this case,

$$
\nu_{r}(R)=\nu_{1}(R)
$$


However, in practice a considerable reduction in the number of function evaluations results in cytolic integration by employing a rule $R$ which uses points on the boundary of the domain. This is because a single function evaluation at such a point provides values for two or more different subdomains. Thus, for such rules,

$$
\nu_{1}\left(R^{(r)}\right)<r^{n} \nu_{1}(R),
$$

and the number of points per subdomain satisfies

$$
\nu_{r}(R)<\nu_{1}(R) .
$$

We define

$$
\bar{\nu}(R)=\lim _{r \rightarrow \infty} \nu_{r}(R) .
$$

In an integration region in which the effect of the boundaries is negligible, it is $\bar{\nu}(R)$ and not $\nu_{1}(R)$ which is the pertinent number of points to consider.

The calculation of $\nu_{r}$ and $\bar{\nu}$ for basic rules and composite rules follows the same lines as the calculation of $\nu_{1}$. In (6.2), if $\alpha_{k}$ (the largest of the $\alpha_{i}$ ) is not equal to one, we have

$$
\nu_{r}(R)=\bar{\nu}(R)=\nu_{1}(R) .
$$

If $\alpha_{k}$ is one, we find

$$
\nu_{r}(\Re)=\left(\frac{r+1}{2 r}\right)^{n_{k}} \nu_{1}(\Re)
$$

and

$$
\bar{\nu}(R)=2^{-n_{k}} \nu_{1}(R) .
$$

Again we find for the composite rule $R$ given by (5.4) that

$$
\nu_{r}(R)=\sum_{\boldsymbol{j}} \nu_{r}\left(R_{j}\right)
$$

and

$$
\bar{\nu}(R)=\sum_{j} \bar{\nu}\left(R_{j}\right) .
$$

$\bar{\nu}$ may be very different from $\nu$. For example, the face centre rule in three dimensions $R_{\mathrm{FC} 3}$ and the centre and vertex rule in three dimensions $R_{\mathrm{Cv} 3}$ are both rules of degree three (see (3.15) and (3.23) above) and both give comparable error estimates. A decision about which to use may rest on the number of points. We find

$$
\nu_{r}\left(R_{\mathrm{FC} 3}\right)=3(r+1) / r
$$

and

$$
\begin{aligned}
\nu_{r}\left(R_{\mathrm{CV} 3}\right) & =\nu_{r}(R(0,0,0))+\nu_{r}(R(1,1,1)) \\
& =1+\left(\frac{r+1}{r}\right)^{3} .
\end{aligned}
$$

In particular,

$$
\begin{aligned}
& \nu_{1}\left(R_{\mathrm{FC} 3}\right)=6, \\
& \nu_{1}\left(R_{\mathrm{Cv} 3}\right)=9,
\end{aligned}
$$

and so over a single domain $R_{\mathrm{FC} 3}$ might be preferred. 
However,

$$
\begin{aligned}
& \bar{\nu}\left(R_{\mathrm{FC} 3}\right)=3, \\
& \bar{\nu}\left(R_{\mathrm{CV} 3}\right)=2,
\end{aligned}
$$

and so over a large subdivided domain the opposite preference might be made. For example, if $r=10$, we find $R_{\mathrm{FC} 3}^{(10)}$ requires 3300 function evaluations and $R_{\mathrm{CV} 8}^{(10)}$ requires 2331 .

This simple example indicates clearly that Gaussian type rules, although superior for holistic integrations, may be very inferior for cytolic integrations. The question of the sharing of points between subdomains and the design of rules which do this are of utmost importance.

This complicating aspect of the point counting means thàt we have to take into consideration for each rule not merely one number $\nu_{1}(R)$, but the function $\nu_{r}(R)$, or at the very least the two numbers $\nu_{1}$ and $\bar{\nu}$.

In Tables I and II the values of $d, \nu, \bar{\nu}$ and $\nu_{r}$ are attached to the corresponding rule. The $n$-dimensional product rule $(R)^{n}$ has values of $\nu_{r}$ satisfying

$$
\nu_{r}\left((R)^{n}\right)=\left(\nu_{r}(R)\right)^{n} \text {. }
$$

Acknowledgment. The author is glad to acknowledge considerable help and advice from Professor John M. Blatt.

Appendix. Properties of Error Coefficients. This Appendix contains certain miscellaneous properties of the error coefficients; most of these properties may be proved using elementary algebra, and their proof is only briefly indicated here.

We may derive a relation by the direct substitution of any function and any rule into (2.23). For example, if we put $f(x)=x^{2 n}$, and use $R=\sum \xi_{j} \cap\left(\alpha_{j}\right)$, we find the set of equations

$$
\frac{\sum \xi_{j} \alpha_{j}^{2 n}}{(2 n) !}=\frac{c_{0}(R)}{(2 n+1) !}+\frac{c_{2}(R)}{(2 n-1) !}+\cdots+\frac{c_{2 n-2}(R)}{3 !}+\frac{c_{2 n}(R)}{1 !} .
$$

\begin{tabular}{|c|c|c|c|c|}
\hline & & $d$ & $\nu_{1}$ & $\bar{\nu}$ \\
\hline $\begin{array}{l}\text { Trapezoidal } \\
\text { One Point }\end{array}$ & $\begin{array}{l}R(1) \\
R(0)\end{array}$ & $\begin{array}{l}1 \\
1\end{array}$ & $\begin{array}{l}2 \\
1\end{array}$ & 1 \\
\hline $\begin{array}{l}\text { Two Point Newton Cotes } \\
\text { Three Point Simpson }\end{array}$ & $\begin{array}{l}R\left(\frac{1}{3}\right) \\
\frac{2}{3} R(0)+\frac{1}{3} R(1)\end{array}$ & $\begin{array}{l}1 \\
3\end{array}$ & $\begin{array}{l}\mathbf{2} \\
\mathbf{3}\end{array}$ & $\begin{array}{l}2 \\
2\end{array}$ \\
\hline $\begin{array}{l}\text { Four Point Newton Cotes } \\
\text { Five Point Newton Cotes }\end{array}$ & $\begin{array}{l}\frac{3}{4} R\left(\frac{1}{3}\right)+\frac{1}{4} R(1) \\
\frac{6}{45} R(0)+\frac{8}{4} R\left(\frac{1}{2}\right)+\frac{7}{45} R(1)\end{array}$ & $\begin{array}{l}3 \\
5\end{array}$ & $\begin{array}{l}4 \\
5\end{array}$ & $\begin{array}{l}3 \\
4\end{array}$ \\
\hline $\begin{array}{l}\text { Two Point Gaussian } \\
\text { Three Point Gaussian }\end{array}$ & $\begin{array}{l}R\left(\sqrt{ } \frac{1}{3}\right) \\
\frac{4}{9} R(0)+\frac{5}{9} R\left(\sqrt{ } \frac{3}{5}\right)\end{array}$ & $\begin{array}{l}3 \\
5\end{array}$ & $\begin{array}{l}2 \\
3\end{array}$ & $\begin{array}{l}2 \\
3\end{array}$ \\
\hline
\end{tabular}

TABLE I

Certain well-known one-dimensional integration rules together with their degree d and the values of $\nu_{1}$ and $\bar{\nu}$. 


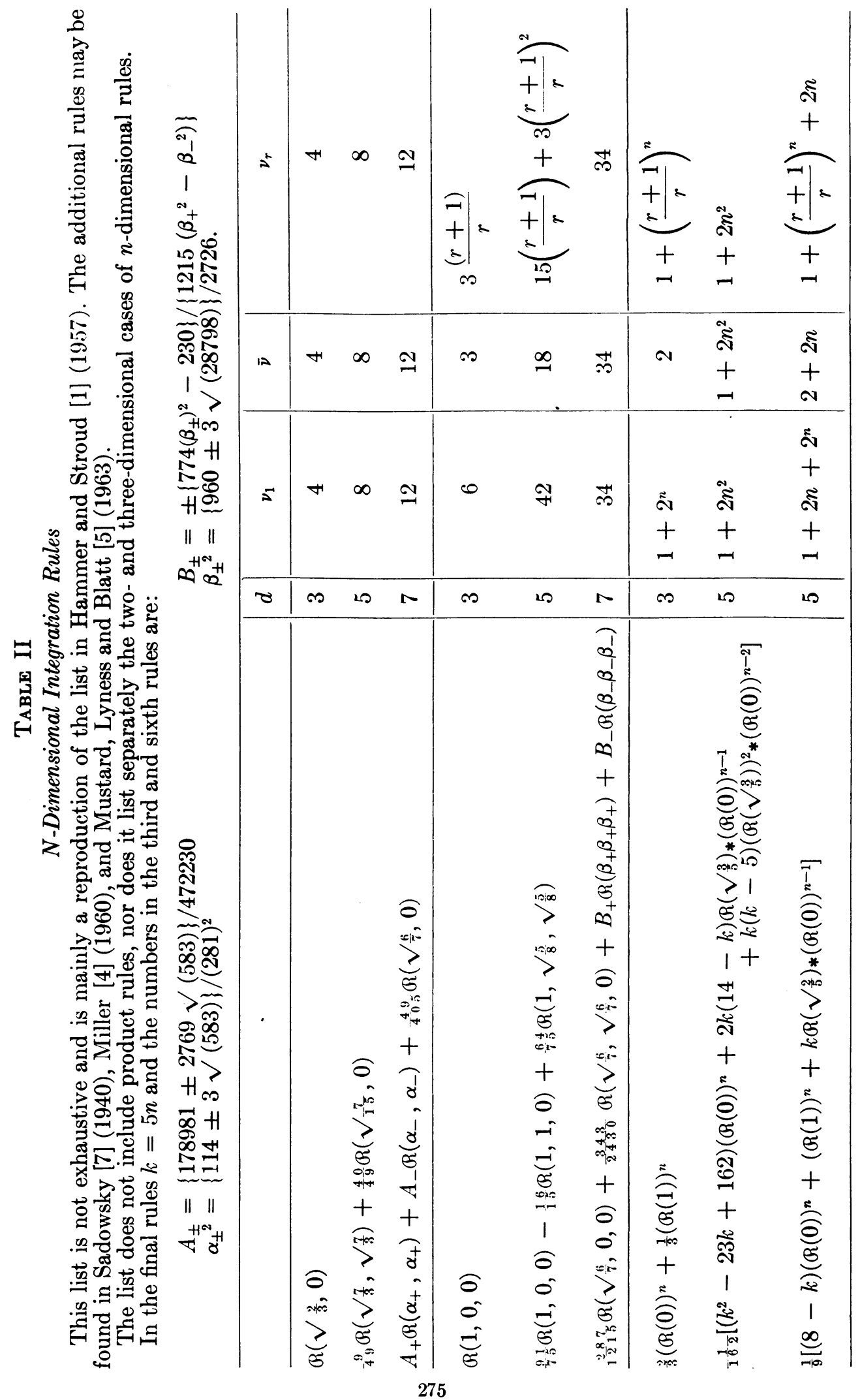


This relation may be used to find successively the error coefficients for a sophisticated composite rule $R$. It is of particular use if the rule is of high degree.

The values of $c_{28}(\alpha)$ for certain values of $\alpha$, namely, $0, \frac{1}{2}$, and 1 , may be simply obtained from the generating function. This is done in Section 2 and the results are listed in (2.11), (2.12), and (2.13). To obtain the numerical values for $\alpha=\frac{1}{3}$ or $\alpha=\frac{2}{3}$ we consider the identity (see Section 4 ):

$$
(\mathbb{R}(1))^{(3)}=\frac{2}{3} R\left(\frac{1}{3}\right)+\frac{1}{3} R(1) .
$$

The error coefficient relation (4.11) and (A.2) above lead to

$$
\begin{aligned}
\left((R(1))^{(3)}\right) & =\frac{1}{3^{2 s}} c_{28}(1) . \\
& =\frac{2}{3} c_{2 s}\left(\frac{1}{3}\right)+\frac{1}{3} c_{28}(1) .
\end{aligned}
$$

Thus

$$
c_{2 s}\left(\frac{1}{3}\right)=\frac{1}{2}\left(-1+\frac{1}{3^{2 s-1}}\right) c_{28}(1),
$$

where $c_{2 s}(1)$ is given by (2.13). A similar procedure leads to

$$
c_{2 s}\left(\frac{2}{3}\right)=\frac{1}{2}\left(-1+\frac{1}{3^{2 s-1}}\right) c_{2 s}(0) \text {. }
$$

The further exploitation of relations such as (A.2) gives relations between error coefficients but not their actual values.

(A.4) and (A.5) may be derived from the generating function (2.10) directly using the identity

$$
2 \cosh \frac{1}{3} \varphi \sinh \frac{1}{3} \varphi=\cosh \frac{1}{3} \varphi \sinh \varphi-\sinh \frac{1}{3} \varphi \cosh \varphi .
$$

It sometimes occurs that we know the error coefficients of an $(n-1)$-dimensional rule $R^{(n-1)}$ and we are interested in the error coefficients of the $n$-dimensional rule $R^{(n-1)} * R$. The relevant result is

$$
c_{2 s_{1} 28_{2} \cdots 2 s_{n}}\left(R^{(n-1)} * R\right)=\frac{1}{n} \sum_{i=1}^{n} c_{2 s_{1} \cdots 2 s_{i-1} 2 s_{i}+1} \cdots 2 s_{n}\left(R^{(n-1)}\right) c_{2 s_{i}}(R) .
$$

This may be proved in the case that $R^{(n-1)}$ and $R$ are basic rules by manipulation of (3.11). The extension to composite rules is trivial.

University of New South Wales

Kensington, N.S.W.

Australia

1. P. C. Hammer \& A. H. Stroud, "Numerical evaluation of multiple integrals. II," $M T A C$, v. 12,1958, pp. $272-280$. MR $21 * 970$.

2. J. N. Lyness \& J. B. B. McHugh, "A progressive procedure," Comput. J., v. 6, 1963/64, pp. 264-270.

3. J. C. P. MiLLER, "Numerical quadrature over a rectangular domain in two or more dimensions. I: Quadrature over a square, using up to sixteen equally spaced points," Math. Comp., v. 14, 1960, pp. 13-20. MR 22 *1075.

4. J. C. P. MILLER, "Numerical quadrature over a rectangular domain in two or more dimensions. II: Quadrature in several dimensions, using special points," Math. Comp., v. 14, 1960, pp. 130-138. MR 22 *6075.

5. D. Mustard, J. N. Lyness \& J. M. Blatt, "Numerical quadrature in n dimensions," Comput. J., v. 6, 1963/64, pp. 75-87. MR $28 * 1762$.

6. D. MustaRd, "Approximate integration in $N$ dimensions," Thesis, Univ. of New South Wales, Australia, 1964.

7. M. SADOWSKY, "A formula for approximate computation of a triple integral," Amer. Math. Monthly, v. 47, 1940, pp. 539-543. MR 2, 62. 